\title{
The Role of Primary and Secondary Infection in Host Response to Plasmodiophora brassicae
}

\author{
Mary Ruth McDonald, Kalpana Sharma, Bruce D. Gossen, Abhinandan Deora, Jie Feng, and Sheau-Fang Hwang
}

First, second, and fourth authors: Department of Plant Agriculture, University of Guelph, Guelph, ON, N1G 2W1 Canada; second, third, and fourth authors: Agriculture and Agri-Food Canada, Saskatoon Research Centre, Saskatoon, SK, S7N 0X2 Canada; fifth and sixth authors: Crop Diversification Centre North, Alberta Agriculture and Rural Development, Edmonton, AB, T5Y 6H3 Canada.

Accepted for publication 7 March 2014.

\begin{abstract}
McDonald, M. R., Sharma, K., Gossen, B. D., Deora, A., Feng, J., and Hwang, S.-F. 2014. The role of primary and secondary infection in host response to Plasmodiophora brassicae. Phytopathology 104:1078-1087.

The disease cycle of Plasmodiophora brassicae consists of a primary phase in root hairs followed by a secondary phase in the root cortex and adjacent tissues. However, the role of root hair infection in subsequent cortical infection and development of $P$. brassicae is not well understood. To examine the role of the primary and secondary stages separately, inoculation studies with resting spores (source of primary zoospores) and secondary zoospores of a virulent and avirulent pathotype were con-

$14.4 \mu \mathrm{m})$ than in previous reports and all were uninucleate. Inoculation with secondary zoospores alone produced both primary and secondary infection, even with the avirulent pathotype. No symptoms developed from inoculation with avirulent primary zoospores but tiny, bead-shaped clubs developed from inoculation with avirulent secondary zoospores. Inoculation with virulent secondary zoospores alone resulted in lower disease severity than inoculation with virulent resting spores alone. The results indicate that recognition of infection by the host and initiation of a response (induction or suppression of resistance) occurs during primary infection, although recognition can also occur during cortical infection and development.
\end{abstract} ducted on canola (Brassica napus). The size of secondary zoospores and number of nuclei were also examined. The zoospores were larger $(\approx 9.6$ to

Plasmodiophora brassicae Woronin, an obligate parasite causing clubroot, is an emerging threat to canola (Brassica napus L.) production in Canada $(21,23)$ and across the Northern Great Plains of the United States. The disease cycle of $P$. brassicae consists of two phases: a primary phase that is restricted to root hairs and occasionally epidermal cells and a secondary phase that involves pathogen infection and proliferation within the root and subsequent symptom development (24). In the primary phase, resting spores (RS) in the soil germinate and release primary zoospores that penetrate root hairs to form primary plasmodia. The primary plasmodia form multinucleate zoosporangia that produce uninucleate secondary zoospores (SZ). This process takes $\approx 5$ days at the optimum temperature of $25^{\circ} \mathrm{C}(39)$. The $\mathrm{SZ}$ are released into the soil solution, infect root cortical cells, and develop into motile secondary plasmodia $(10,27)$. After a mobile phase within the root, the young plasmodia coalesce into sessile vegetative plasmodia that divide to form long-lived RS $(10,24$, 27). Cortical infection results in hyperplasia and hypertrophy of the root cells, which produces the characteristic swollen, rounded clubbed root symptoms $(24,27)$. Clubbed roots are readily visible within 5 to 6 weeks of inoculation at temperatures of 20 to $25^{\circ} \mathrm{C}$ (40).

The roles of primary and secondary infection in pathogenesis by $P$. brassicae are not fully understood. Primary zoospores and SZ cannot be differentiated visually; both are ovate, with two whiplash flagella of unequal length $(12,14,24)$. Some authors and at least one authoritative pathology textbook have concluded that SZ fuse to form binucleate zoospores prior to infecting the root

Corresponding author: M. R. McDonald; E-mail address: mrmcdona@uoguelph.ca

http://dx.doi.org/10.1094/PHYTO-07-13-0189-R

(C) Her Majesty the Queen in Right of Canada, as represented by the Minister of Agriculture and Agri-Food Canada.
Additional keyword: clubroot. cortex $(2,10,24)$ but observation of fusion has not been reported (10). Thus, it is still unclear whether fusion of SZ is a necessary step prior to cortical infection.

Inoculation with RS initially produces only primary (root hair) infection but inoculation with SZ results in both primary and secondary infection in susceptible hosts $(14,35)$. Primary infection occurs in both susceptible and resistant host cultivars $(6,9,15,34)$. Primary infection can also occur in several nonhost species (34) but secondary infection and development of small numbers of RS have been reported in only a few species that are generally considered nonhosts (33).

It is not known whether primary infection affects the initiation of resistance in host species. However, recent studies indicate that nonhost resistance to secondary infection is induced initially during root hair infection (RHI). Inoculation of a nonhost, perennial ryegrass (Lolium perenne L.) with SZ resulted in secondary infection and production of young plasmodia but not mature plasmodia or RS (14).

The predominant pathotypes of $P$. brassicae in Canada are pathotypes 2, 3, 5, and 6, based on the differential set of Williams (45) and confirmed using the European Clubroot Differential set $(42,43)$. Several pathotypes of $P$. brassicae are present on the Canadian prairies, and more than one pathotype can be present in a field $(3,43,46)$. However, pathotype 3 (P3) is the predominant pathotype found on canola in the region (3). Breakdown of resistance to $P$. brassicae has been documented in numerous Brassica spp. (8,32). Loss of genetic resistance is a concern for Canadian canola producers $(23,32)$, where resistance represents one of the most effective tools to manage clubroot (18).

The current study was conducted to assess the roles of infection by primary and SZ in the initiation of susceptibility and resistance to $P$. brassicae in canola. Experiments were conducted to compare both compatible (susceptible host) and incompatible (resistant host) reactions of canola plants inoculated with primary 
or SZ of virulent and avirulent pathotypes of $P$. brassicae. The treatments were selected to permit evaluation of two competing hypotheses of disease reaction to clubroot. One hypothesis was that primary infection suppresses initiation of resistance in a susceptible cultivar and stimulates initiation of resistance in a resistant cultivar. The alternative was that primary infection has no effect on initiation of resistance. In addition, flagellate and encysted SZ were examined to determine whether they were unior binucleate and to confirm their morphology.

\section{MATERIALS AND METHODS}

Plant material, inoculum, and inoculation. 'Zephyr' canola (AAFC, Saskatoon, SK, Canada) was selected for the study because it is resistant to pathotype 6 (P6) of P. brassicae but susceptible to P3 (A. Deora, unpublished data). All plants were watered daily with demineralized water adjusted to $\mathrm{pH} 6.3$ using commercial vinegar (5\% acetic acid), and fertilized weekly with a nutrient solution composed of $0.025 \%$ each of NPK (20:20:20; Plant Products Co. Ltd., Canada).

A field isolate of $\mathrm{P} 3$ was obtained from clubbed roots of canola grown in a commercial field near Edmonton, AB, Canada, where P3 is predominant $(42,43)$. An isolate of P6 (S. Strelkov, personal communication) was obtained from clubbed roots of canola grown at the Muck Crops Research Station of the University of Guelph, Holland Marsh, ON, Canada. Inoculum of each pathotype was increased prior to the study on the highly susceptible Shanghai pak choy 'Mei Qing Choy' (Stokes Seeds, St. Catharines, ON, Canada) under controlled conditions (38). The clubbed roots were stored at $-20^{\circ} \mathrm{C}$ prior to use.

Inoculum of RS was prepared based on the method of Jones et al. (25). Briefly $\approx 3 \mathrm{~g}$ of frozen clubs (swollen clubbed roots) were thawed at room temperature and homogenized in $100 \mathrm{ml}$ of water at high speed for $2 \mathrm{~min}$, and the resulting spore suspension was strained through eight layers of cheesecloth. The RS concentration was determined using a hemocytometer and adjusted to $3 \times 10^{5}$ spores $\mathrm{ml}^{-1}$. Freshly prepared inoculum was used for each inoculation. Five-day-old seedlings were inoculated by pipetting $5 \mathrm{ml}$ of the spore suspension at the base of each seedling and control plants were mock inoculated with deionized water.

Inoculum of SZ for an initial trial in cell culture plates was produced using the method of Feng et al. (14), with only slight modification. Seed was planted in 2-by-20-by-10-cm plastic trays filled with washed, autoclaved coarse sand. Seven-day-old seedlings were inoculated with $500 \mathrm{ml}$ of $1 \times 10^{8} \mathrm{RS} \mathrm{ml}^{-1}$. To avoid cross-contamination, separate trays were used for each pathotype. After 7 days, $\approx 4,000$ plants were uprooted and the roots were washed with tap water. The foliage was then cut off near the hypocotyl, and the roots were further rinsed three times by shaking at $150 \mathrm{rpm}$ for $20 \mathrm{~min}$ in $200 \mathrm{ml}$ of sterile deionized water in a 500 -ml flask. The roots $(\approx 1,000$ per flask) were shaken for $20 \mathrm{~h}$ in $50 \mathrm{ml}$ of deionized water at $100 \mathrm{rpm}$ to stimulate the release of SZ. After removing the roots, $10 \mathrm{ml}$ of the zoospore suspension was concentrated by centrifugation at $5,000 \times g$ for $5 \mathrm{~min}$ and adjusted to $1 \times 10^{6}$ zoospores $\mathrm{ml}^{-1}$. Ten samples of this suspension were examined microscopically to confirm that no RS were present in the suspension. The concentration of SZ in the initial inoculum suspension was estimated using a hemocytometer and adjusted to $3 \times 10^{5}$ spores $\mathrm{ml}^{-1}$. The spore suspension was then used immediately for inoculation.

For the study of symptom development, SZ were produced on canola seedlings planted in Conetainers (plastic 21-by-3.8-cm cones, Conetainers; Stuewe and Sons Inc., Corvallis, OR) filled with sand, thinned to five seedlings per cone, and inoculated with $5 \mathrm{ml}$ of $1 \times 10^{8} \mathrm{RS} \mathrm{ml}^{-1}$ per cone at 5 days after seeding (DAS). The seedlings were washed and then planted directly into treatment cones as a source of SZ at 7 days after the initial date of inoculation with RS (12 DAS) (35). This approach was used because it was not possible to harvest the large number of SZ required using the initial methodology.

Primary and secondary infection. Canola seed was sown into 24-well cell culture plates (Sigma-Aldrich Canada Ltd., Oakville, ON, Canada) filled with sand. The plates were watered daily and maintained in a growth room at 20 and $25^{\circ} \mathrm{C}$ (night and day, respectively) with $75 \%$ relative humidity and a $16-\mathrm{h}$ photoperiod. After 5 days, the plates were thinned to one seedling per cell. Each experimental unit consisted of a single seedling, and the study was arranged in a randomized complete block design (RCBD), with four replicates at each of three sampling dates. There were nine inoculation treatments: (i) RS of P3, (ii) RS of P6, (iii) RS-P3 at 10 DAS, (iv) RS-P6 at 10 DAS, (v) SZ of P3, (vi) SZ-P6, (vii) RS-P3 + SZ-P3, (viii) RS-P6 + SZ-P3, and (ix) a control inoculated with sterile water only. All of the RS treatments except those designated as "at 10 DAS" were applied to 5-day-old seedlings. SZ treatments and the late (at 10 DAS) RS treatments were applied to 10-day-old seedlings. Each seedling was inoculated by pipetting $0.5 \mathrm{ml}$ of $3 \times 10^{5}$ spores $\mathrm{ml}^{-1}$ at the base of the seedling. At optimum temperature for pathogen development, the primary phase of development is nearly complete and $\mathrm{SZ}$ are released at $\approx 5$ days after inoculation $(38,40)$. Therefore, SZ were applied 5 days later than the primary zoospores to ensure that, as much as possible, secondary infection in all of the treatments occurred at the same time, and that the seedlings were at a uniform developmental stage when secondary infection occurred. The late inoculation treatments with RS were included to provide an inoculated control for treatments at 10 DAS.

Four seedlings per treatment were destructively sampled at 11 , 13, and 15 DAS (1, 3, and 5 days after application of the SZ and late application of RS). The root of each seedling was separated from the foliage, washed in running water, and placed individually in 2-ml centrifuge tubes containing a fixative solution of $50 \%$ ethanol. Five fields of view along a 2 -cm-long root segment from each taproot were assessed using a compound microscope with $\mathrm{a} \times 10$ objective lens (40). In each field of view, the incidence of RHI and secondary plasmodia in cortical tissue were counted based on the method of Feng et al. (14).

Symptom development. Seed of Zephyr canola were planted in Conetainers filled with soilless mix (Sunshine mix number 4; Sun Gro Horticulture Canada Ltd., Spruce Grove, AB, Canada). A 5 -ml pipette tip was inserted into the soilless mix in the center of the pot. The seedlings were thinned to one per pot after 5 days. The same nine treatments described previously were examined in an RCBD with four replicates and 10 plants per replicate. The main group of RS suspension treatments $\left(5 \mathrm{ml}, 3 \times 10^{5}\right.$ spores $\left.\mathrm{ml}^{-1}\right)$ were applied to 5-day-old seedlings and SZ treatments were applied to 10-day-old seedlings, as in the initial trial. However, SZ were applied by removing the 5-ml pipette tip from each pot and planting five infected seedlings into the cavity created by the pipette tip (36). Noninoculated plants were used as a control. The seedlings used for inoculation were removed after 5 days.

The plants were harvested 52 DAS, which was 42 days (6 weeks) after inoculation with SZ. Plant height above the hypocotyl was measured and the roots were washed and assessed for clubroot incidence and severity, based on visual symptoms of root clubbing. The plants were separated into classes using a standard 0-to-3 scale, where $0=$ no clubbing, $1<$ one-third of the root with symptoms of clubbing, 2 = one-thirds to two-thirds clubbed, and $3>$ two-thirds clubbed (31). A disease severity index (DSI) was calculated using the following equation (43): DSI $=(\Sigma[$ (class number)(number of plants in each class) $]) /[$ (total number plants per sample)(number classes -1$)] \times 100$.

Each root was then prepared, sectioned, and stained, and the intensity of cortical infection was assessed using the method of Sharma et al. (40). A segment (0.5 cm long) was cut from the top 0 to $1 \mathrm{~cm}$ of each taproot and dehydrated through an ethanol series, and a $4-\mu \mathrm{m}$-thick cross-section was cut using a microtome. 
The sections (one per root, four per treatment) were placed on a microscope slide and stained in $0.5 \%$ methylene blue for $5 \mathrm{~min}$. Development of $P$. brassicae was assessed based on the presence of key developmental stages: (i) secondary plasmodia, (ii) vegetative plasmodia, and (iii) RS. The number of cells that contained plasmodia or RS in each microscope field ( $\times 10$ objective lens) were counted in five fields of view per section. Cortical infection was assessed using image analysis, by estimating the area $(\%)$ occupied by plasmodia and RS in digital images of each field of view ( $\times 10$ objective lens) using image analysis software (Assess version 2.0; American Phytopathological Society, St. Paul, MN). In total, 20 fields of view were assessed per treatment in the trial. The trial was repeated.

To observe the location and extent of lignification, another set of $4-\mu \mathrm{m}$-thick cross-sections (one per root, four per treatment) was placed on a microscope slide and stained with $1 \%$ toluidine blue $\mathrm{O}$ for $3 \mathrm{~min}$. These roots were examined using a compound microscope following the method of Deora et al. (7). Lignified areas stained blue and pectic substances stained pink-purple (36).

Infectivity of RS from bead-shaped clubs. A study was conducted to determine whether the RS produced in bead-shaped clubs were infectious. In those treatments where bead-shaped clubs developed, the tap root and lateral roots were collected, washed, diced into small pieces, and used to inoculate 5-day-old seedlings, with pieces from two roots applied to each seedling. Roots of noninoculated seedlings (negative control) and plants that had been inoculated with RS of P6 (positive control) were also assessed. The inoculated plants were harvested 6 weeks after transplanting and assessed for clubroot incidence and severity as described previously. The trial was laid out in an RCBD with four replicates and 10 plants per replicate. The trial was repeated.

Nuclear visualization. The nuclei of zoospores forming within root hairs were stained and photographed. Seedlings of the susceptible canola '46A76' (Pioneer Hi-Bred, Caledon, ON, Canada) were grown at 24 and $20^{\circ} \mathrm{C}$ in Conetainers filled with autoclaved $\left(121^{\circ} \mathrm{C}\right.$ for $\left.30 \mathrm{~min}\right)$ sand. A $5-\mathrm{ml}$ suspension of $1 \times 10^{6}$ $\mathrm{RS} \mathrm{ml} \mathrm{m}^{-1}$ of $\mathrm{P} 6$, prepared as described previously, was applied at the base of each 8-day-old seedling; the control received water only. There were three replications with three plants per replicate. Plants were watered every day as required. The roots were harvested at 12 days after inoculation and preserved in ethanol/acetic acid (3:1) solution. The harvested roots were stained with Hoechst 33258 (bisbenzimide H; Sigma-Aldrich Canada Ltd.) at $20 \mu \mathrm{g} / \mathrm{ml}$ and observed under an upright Leica DM 6000B confocal laser scanning microscope (CLSM) (Leica Microsystems, Concord, ON Canada). Only root hairs that contained the zoosporangial stages of primary infection were targeted to observe the nuclei of SZ. An excitation wavelength of $405 \mathrm{~nm}$ and an emission and detection channel of 427 to $490 \mathrm{~nm}$ were used. To produce a fluorescence image, averaging (oversampling) was conducted. For Z-sectioning, 10 to 30 sections were obtained, depending on the depth of the sample. For the overlaid image of fluorescence and differential interference contrast presented, the thickness of the sample was $35 \mu \mathrm{m}$, for which 85 sections were obtained at a step size of $0.42 \mu \mathrm{m}$.

To stain the nuclei of flagellate zoospores, $100 \mu \mathrm{l}$ of SZ suspension (produced as described previously) was placed on a lysine-coated, glass-bottomed culture dish (14 $\mathrm{mm}$ in diameter; MatTek Corporation, MA). The zoospores were fixed by adding $100 \mu \mathrm{l}$ of $2 \%$ glutaraldehyde. Zoospores were allowed to settle on the bottom of the dish for $30 \mathrm{~min}$, rinsed three times in phosphate buffer ( $\mathrm{pH}$ 6.8) at 2-min intervals, stained for 1 min with $4^{\prime}-6$ diamidino-2-phenylindole at $1 \mu \mathrm{g} / \mathrm{ml}$ of water (Sigma-Aldrich Canada Ltd.), rinsed with buffer, and mounted on a glass slide in $50 \%$ glycerol with $0.1 \%$ p-phenylendiamine. The number of nuclei in each zoospore was assessed using epifluorescence microscopy with a blue filter (wide-band UV), excitation band pass of 360 to $385 \mathrm{~nm}$ (dichromatic beam splitter DM400), and emission barrier filter of $420 \mathrm{~nm}$ (Olympus BX60F5 microscope; Olympus Optical Co. Ltd.).

For nuclear staining of encysted zoospores, $5 \mathrm{ml}$ of the SZ suspension was concentrated by centrifugation at $5,000 \times g$ for $5 \mathrm{~min}$, then assessed as described above. At least 25 flagellate or encysted zoospores were observed in each assessment and the study was repeated.

The size of SZ produced and held in various solutions was assessed. After harvesting the roots for SZ production as described above, roots were incubated in a shaker in either deionized water, phosphate buffer $\left(\mathrm{Na}_{2} \mathrm{HPO}_{4}\right.$ and $\left.\mathrm{NaH}_{2} \mathrm{PO}_{4}\right)$ (SigmaAldrich, St. Louis), or sodium chloride physiological solution (Sigma-Aldrich). The $\mathrm{pH}$ of the phosphate buffer and physiological saline was adjusted to 7.0 with $\mathrm{NaOH}$ (Sigma-Aldrich). After $20 \mathrm{~h}$ of incubation, a suspension of swimming or encysted zoospores (obtained by vortexing for $1 \mathrm{~min}$ ) from each solution was fixed with $1 \%$ glutaraldehyde. The length and width of 30 fixed zoospores from each solution were measured on photos taken using a stereo binocular microscope (Nikon Eclipse 5.1; Nikon Corporation, Japan).

Statistical analysis. There were two repetitions of each experiment investigating infection and symptom development. A general linear model analysis of variance was conducted using Proc GLM of SAS (version 9.1; SAS Institute, Inc., Cary, NC). Prior to analysis, RHI (\%), clubroot incidence (\%), clubroot severity (DSI), and area of cortical infection (\%) data were arcsine transformed to improve the normality and homogeneity of variance but nontransformed means are presented for uniformity of presentation. There was no repetition effect or repetitiontreatment interaction for any response variable; therefore, the repetitions of each trial were pooled for subsequent analysis. Means separation was conducted using Tukey's test at $\alpha \leq 0.05$. The correlation between cortical colonization and DSI was examined using Pearson's correlation coefficient in Proc Corr of SAS.

In the infection and symptom development trials, a few specific comparisons were particularly important for hypothesis testing. Comparison of RS-P6 with SZ-P6 provided a measure of the role of primary infection in initiation of resistance to an avirulent pathotype. Similarly, comparison of RS-P3 with SZ-P3 provided a measure of the role of primary infection in suppression of resistance to a virulent pathotype. Comparison of RS-P6 + SZ-P3 versus RS-P3 + SZ-P3 provided a measure of the role of early initiation of an incompatible host reaction (P6) or a compatible reaction (P3) prior to secondary infection and subsequent development of the virulent pathotype (SZ-P3). Similarly, comparison of RS-P6 + SZ-P3 with SZ-P3 provides a measure of the impact of early initiation of resistance on subsequent development of a virulent pathotype, and comparison of RS-P3 + SZ-P3 with SZP3 provides a measure of the impact of early suppression of resistance on subsequent development of a virulent pathotype.

\section{RESULTS}

Primary infection. Each of the inoculation treatments resulted in RHI (Table 1). At 13 and 15 DAS (3 and 5 days after inoculation), RHI was higher following inoculation with SZ-P3 compared with SZ-P6. Substantial levels of infection were first observed at 1 day after inoculation in plants inoculated with RSP3 + SZ-P3 (63\%), RS-P3 (46\%), and RS-P6 + SZ-P3 (35\%). At 15 DAS (5 days after inoculation), RHI was relatively high for all of the treatments except RS-P6 at 10 DAS (26\%), although differences among treatments were still evident. RHI was higher for RS-P3 than RS-P6 and higher for SZ-P3 than SZ-P6. RHI with RS-P3 + SZ-P3 was higher than RS-P3 alone. However, RHI was higher with RS-P3 + SZ-P3 than RS-P6 + SZ-P3.

The incidence of RHI increased over time. There was a small inoculation treatment-sampling date interaction $(F=148.5, P=$ 
0.0001, compared with $F=5,308$ for sampling date and 2,249 for treatment) but inoculation treatment generally continued to exhibit a pattern of response at the later assessment dates similar to that observed at 11 DAS.

Secondary infection. The most important differences in this study were observed in infection of the root cortex (Fig. 1) and subsequent symptom expression (Table 2). Substantial numbers of plasmodia in the root cortex were observed at 11 DAS in plants inoculated with RS-P3 + SZ-P3 (15 secondary plasmodia) and RS-P3 (10 secondary plasmodia). Plasmodia were first observed at 13 DAS in plants inoculated with RS-P3 at 10 DAS and SZ-P6, and at 15 DAS in plants inoculated with RS-P6 at 10 DAS. Plasmodia produced from primary and SZ were similar in morphology (Fig. 2A and B).

By 15 DAS, the differences among treatments were even more distinct than at the earlier assessment dates (Fig. 1). As expected, inoculation with RS-P3 resulted in many more secondary plasmodia per field of view (mean of 24 plasmodia per field) than RSP6 (12 plasmodia). Inoculation with SZ alone resulted in fewer plasmodia than RS of the same pathotype; 12 plasmodia for RSP6 versus 9.5 plasmodia for SZ-P6, and 24 plasmodia for RS-P3 versus 16 plasmodia for SZ-P3. RS-P3 + SZ-P3 resulted in more plasmodia (30 plasmodia) than either SZ-P3 alone (15 plasmodia) or RS-P3 alone (24 plasmodia). RS-P6 + SZ-P3 resulted in fewer plasmodia than RS-P3 + SZ-P3 (15 versus 30). There were no differences in the number of plasmodia when SZ-P3 was applied alone or in combination with RS-P6.

Symptom development. The pattern of symptom development and the extent and development of cortical infection were evaluated at 52 DAS. The area of cortical infection, clubroot severity, and the number of cells containing RS all showed the same pattern of response to inoculation treatment (Table 2). The area of cortical colonization and the DSI were highly correlated $(r=0.92, P<0.0001)$. These trends were similar to that of early (15 DAS) cortical infection above, except that no symptoms developed on plants inoculated with RS-P6, even though some plasmodia had developed by 15 DAS and young plasmodia were still visible at 47 DAS (22 infected cells per field of view).

In both the compatible and incompatible interactions, inoculation with RS elicited a different pattern of response than inoculation with SZ (Table 2). Inoculation with RS-P3 resulted in the highest values for both area of cortical infection (33\%) and number of cells with RS (32 cells), and $100 \%$ clubroot incidence and severity. SZ-P3 produced less cortical infection (12\%) and lower incidence and severity (78 and 67\% DSI, respectively) than

TABLE 1. Incidence of primary infection (root hair infection) on canola 'Zephyr' resulting from inoculation with resting spores (RS) and secondary zoospores (SZ) of pathotypes 3 and 6 (P3 and P6, respectively) of Plasmodiophora brassicae, assessed at 11, 13, and 15 days after seeding (DAS) $(1,3$, and 5 days after inoculation with secondary zoospores)

\begin{tabular}{lccc}
\hline & \multicolumn{3}{c}{ Root hair infection $(\%)^{\mathrm{z}}$} \\
\cline { 2 - 4 } Spore type, pathotype & $11 \mathrm{DAS}$ & $13 \mathrm{DAS}$ & $15 \mathrm{DAS}$ \\
\hline RS-P6 (10 DAS) & $2 \mathrm{a}$ & $15 \mathrm{a}$ & $26 \mathrm{a}$ \\
RS-P3 (10 DAS) & $3 \mathrm{a}$ & $26 \mathrm{~b}$ & $48 \mathrm{~b}$ \\
SZ-P6 & $4 \mathrm{a}$ & $32 \mathrm{c}$ & $55 \mathrm{c}$ \\
SZ-P3 & $10 \mathrm{a}$ & $47 \mathrm{e}$ & $74 \mathrm{e}$ \\
RS-P6 & $22 \mathrm{~b}$ & $41 \mathrm{~d}$ & $50 \mathrm{~b}$ \\
RS-P6+SZ-P3 & $35 \mathrm{c}$ & $55 \mathrm{f}$ & $60 \mathrm{~d}$ \\
RS-P3 & $46 \mathrm{~d}$ & $64 \mathrm{~g}$ & $72 \mathrm{e}$ \\
RS-P3+SZ-P3 & $63 \mathrm{e}$ & $73 \mathrm{~h}$ & $80 \mathrm{f}$ \\
Standard error & 0.44 & 1 & 0.7 \\
\hline
\end{tabular}

y The 10 DAS treatments were inoculated with RS spores at the same time as the SZ treatments were applied. All other RS treatments were applied 5 DAS.

${ }^{z}$ Values are the means of eight replications, with one plant per rep. Means within a column followed by the same letter do not differ at $P<0.05$ based on Tukey's test.
RS-P3. The opposite pattern was observed in the incompatible interaction; inoculation with RS-P6 produced $0 \%$ clubroot incidence (no symptoms) but inoculation with SZ-P6 produced low levels of cortical infection (4\%) and development of RS (2 cells). Clubroot symptoms developed following inoculation with SZ-P6, with a low incidence and severity consisting solely of small, bead-shaped clubs.

RS-P3 + SZ-P3 produced $100 \%$ incidence and severity and the highest cortical infection and number of cells with RS, the same as RS-P3 alone (Table 2). However, RS-P6 + SZ-P3 resulted in reduced levels of pathogen development in the root cortex: incidence and severity were lower (85 and $86 \%$ DSI, respectively), as was the area of cortical infection (18\%) and number of cells with RS (26 cells). The only unusual result was the comparison of SZ-P3 with RS-P6 + SZ-P3. Inoculation with RS-P6 + SZ-P3 resulted in higher area of cortical infection, more RS, and higher clubroot severity than SZ-P3 alone.

No clubs developed on plants inoculated with RS-P6 or on the noninoculated control (Table 2). Typical large clubs developed on all of the other treatments. The only exception was SZ-P6, where tiny, bead-shaped clubs developed in strings or clusters along the root (Fig. 2C to E). RS were present in the infected cortical cells of the bead-shaped clubs (Fig. 3F). In all, $\approx 20$ plants were assessed after an additional 2 weeks of growth ( 8 weeks after inoculation), and the size and shape of the clubs had not changed.

Plant height and shoot weight were generally highest, and root mass and clubroot incidence and severity were lowest, in plants with no clubbing (inoculated with RS-P6 or controls) or with

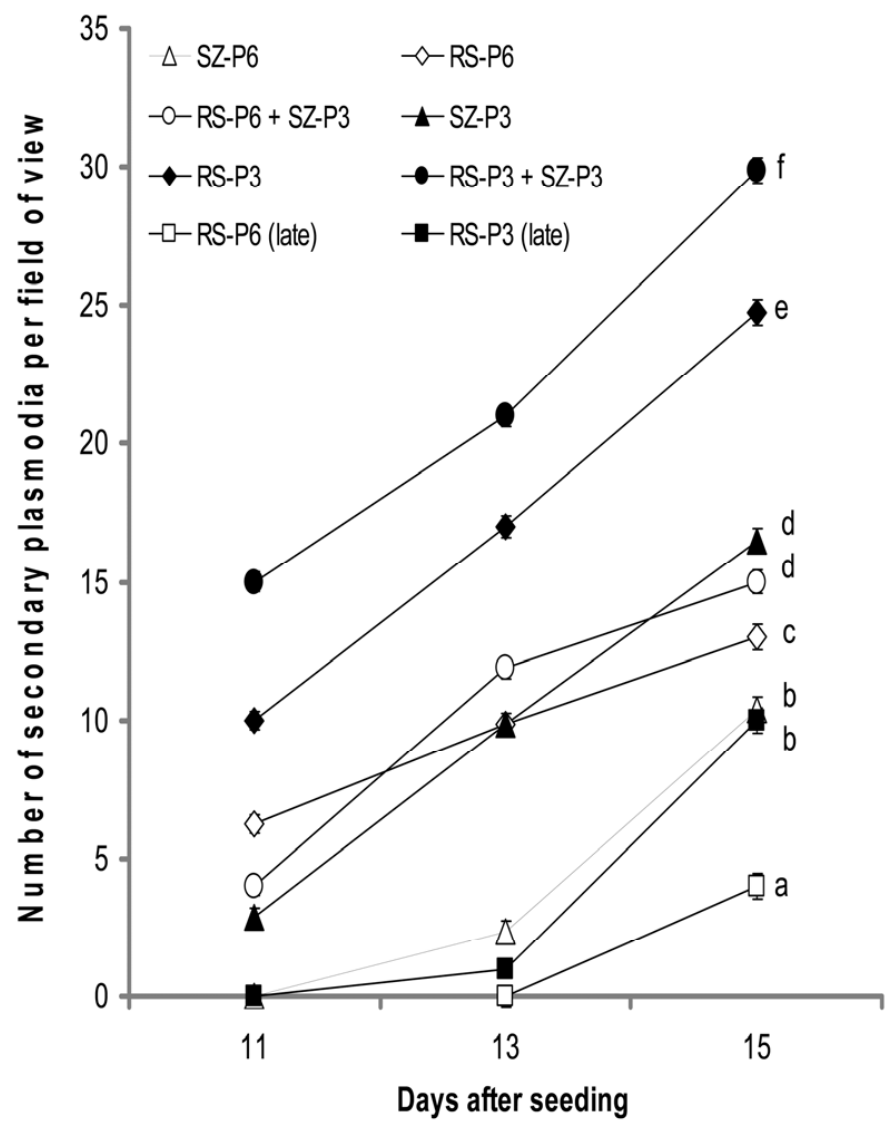

Fig. 1. Secondary infection (number of plasmodia per field of view) of canola 'Zephyr' at 1, 3, and 5 days after inoculation with resting spores (RS) or secondary zoospores (SZ). Regression slopes among the treatments were similar and, therefore, are not presented. Data points are the means of observed values of eight replications (one plant per rep). Means at 5 days after inoculation with the secondary zoospores followed by the same letter do not differ based on Tukey's test at $P \leq 0.05$. Capped lines $=$ standard error. 
bead-shaped clubs (SZ-P6), intermediate with RS-P6 + SZ-P3 and SZ-P3, and lowest and highest in plants with large clubs (RSP3 and RS-P3 + SZ-P3) (Table 2). Similarly, the root biomass of plants inoculated with RS-P6 (1.1 g) and SZ-P6 (1.4 g) were similar but only SZ-P6 produced clubroot symptoms, although the bead-shaped clubs were considerably smaller in size and weight than typical clubs (Table 2).

Proliferation of $P$. brassicae resulted in severe distortion of root growth and development (Fig. 3). In cross-sections of the roots of control plants, the periderm, cortex, and stele region were organized and continuous (Fig. 3A and B). In plants inoculated with RS-P6, young plasmodia were observed in the cortical cells and stele but the pathogen did not develop further (Fig. 3C and D). As a result, plants inoculated with RS-P6 did not differ morphologically from the controls. Typical clubs developed from inoculation with RS-P3, RS-P3 + SZ-P3, SZ-P3, and RS-P6 + SZ-P3 but the clubs from inoculation with SZ-P3 and RS-P6 + SZ-P3 were smaller than normal. In all of the typical clubs, cortical cells of the infected roots became enlarged and intermingled with the secondary phloem, and the distinct organization of tissues into cortex, endodermis, and stele was lost (Fig. 3G and H). The area occupied by cells with walls that stained for lignin in the stele decreased rapidly during pathogen colonization (Fig. $3 \mathrm{G}$ and $\mathrm{H}$ ).

In the bead-shaped clubs, cortical cells were highly plasmolyzed and the stele was continuous (Fig. 3E and F). The pathogen did not penetrate into the stele. As a result, there was limited cell proliferation in these tissues (Fig. 3F). Plants inoculated with RS from the bead-shaped clubs became heavily infected and produced large clubs.

Nuclear visualization. Zoosporangia were present in most of the infected root hairs at 12 days after inoculation. In mature zoosporangia, the plasmodial cytoplasm was cleaved to produce individual zoosporangia (Fig. 4A). CLSM observation showed that these zoosporangia were multinucleate and each nuclei reflected production of uninucleate incipient SZ within the root hairs. Depending on the size, one to six nuclei were present in a

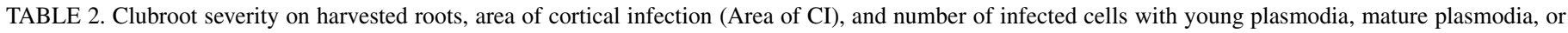

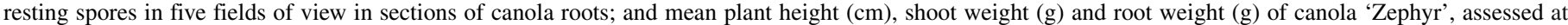

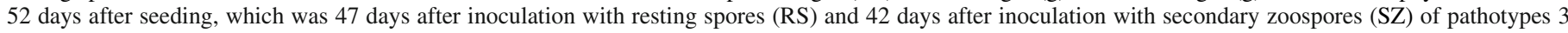
and 6 (P3 and P6, respectively) of Plasmodiophora brassicae ${ }^{\mathrm{Z}}$

\begin{tabular}{|c|c|c|c|c|c|c|c|c|c|}
\hline \multirow[b]{2}{*}{$\begin{array}{l}\text { Spore type, } \\
\text { pathotype }\end{array}$} & \multicolumn{6}{|c|}{ Number of infected cells } & \multicolumn{3}{|c|}{ Plant growth parameters } \\
\hline & $\begin{array}{c}\text { Incidence } \\
(\%)\end{array}$ & $\begin{array}{c}\text { Severity } \\
(\%)\end{array}$ & $\begin{array}{c}\text { Area of CI } \\
(\%)\end{array}$ & $\begin{array}{c}\text { Young } \\
\text { plasmodia }\end{array}$ & $\begin{array}{c}\text { Mature } \\
\text { plasmodia }\end{array}$ & $\begin{array}{c}\text { Resting } \\
\text { spores }\end{array}$ & $\begin{array}{l}\text { Plant height } \\
(\mathrm{cm})\end{array}$ & $\begin{array}{c}\text { Shoot weight } \\
(\mathrm{g})\end{array}$ & $\begin{array}{c}\text { Root weight } \\
(\mathrm{g})\end{array}$ \\
\hline RS-P6 & $0 \mathrm{a}$ & $0 \mathrm{a}$ & $0.1 \mathrm{a}$ & $22 \mathrm{~d}$ & $0 \mathrm{a}$ & $0 \mathrm{a}$ & $49 \mathrm{c}$ & $4.0 \mathrm{c}$ & $1.1 \mathrm{a}$ \\
\hline SZ-P6 & $67 \mathrm{~b}$ & $31 \mathrm{~b}$ & $4 \mathrm{~b}$ & $13 \mathrm{c}$ & $6 \mathrm{~b}$ & $2 b$ & $54 \mathrm{c}$ & $4.0 \mathrm{c}$ & $1.4 \mathrm{a}$ \\
\hline SZ-P3 & $78 \mathrm{c}$ & $67 \mathrm{c}$ & $12 \mathrm{c}$ & $6 \mathrm{~b}$ & $12 \mathrm{~d}$ & $12 \mathrm{c}$ & $28 \mathrm{~b}$ & $3.0 \mathrm{~b}$ & $2.2 \mathrm{~b}$ \\
\hline RS-P6 + SZ-P3 & $85 \mathrm{c}$ & $84 d$ & $18 \mathrm{~d}$ & $1 \mathrm{a}$ & $15 \mathrm{e}$ & $26 \mathrm{~d}$ & $28 \mathrm{~b}$ & $2.6 \mathrm{~b}$ & $2.2 \mathrm{~b}$ \\
\hline RS-P3 & $100 \mathrm{~d}$ & $100 \mathrm{e}$ & $33 \mathrm{e}$ & $1 \mathrm{a}$ & $10 \mathrm{c}$ & $32 \mathrm{e}$ & $15 \mathrm{a}$ & $1.3 \mathrm{a}$ & $3.6 \mathrm{c}$ \\
\hline RS-P3 + SZ-P3 & $100 \mathrm{~d}$ & $100 \mathrm{e}$ & $34 \mathrm{e}$ & $1 \mathrm{a}$ & $9 \mathrm{c}$ & $32 \mathrm{e}$ & $10 \mathrm{a}$ & $1.0 \mathrm{a}$ & $4.2 \mathrm{c}$ \\
\hline Control & 0 & 0 & 0 & 0 & 0 & 0 & $50 \mathrm{c}$ & $4.6 \mathrm{c}$ & $1.1 \mathrm{a}$ \\
\hline Standard error & 0.97 & 1.8 & 0.40 & 0.44 & 0.48 & 0.60 & 1.1 & 0.68 & 0.97 \\
\hline
\end{tabular}

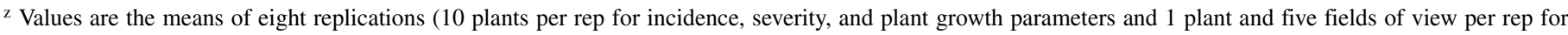
data on number of infected cells). Means in the columns followed by the same letter do not differ based on Tukey's test at $P \leq 0.05$.
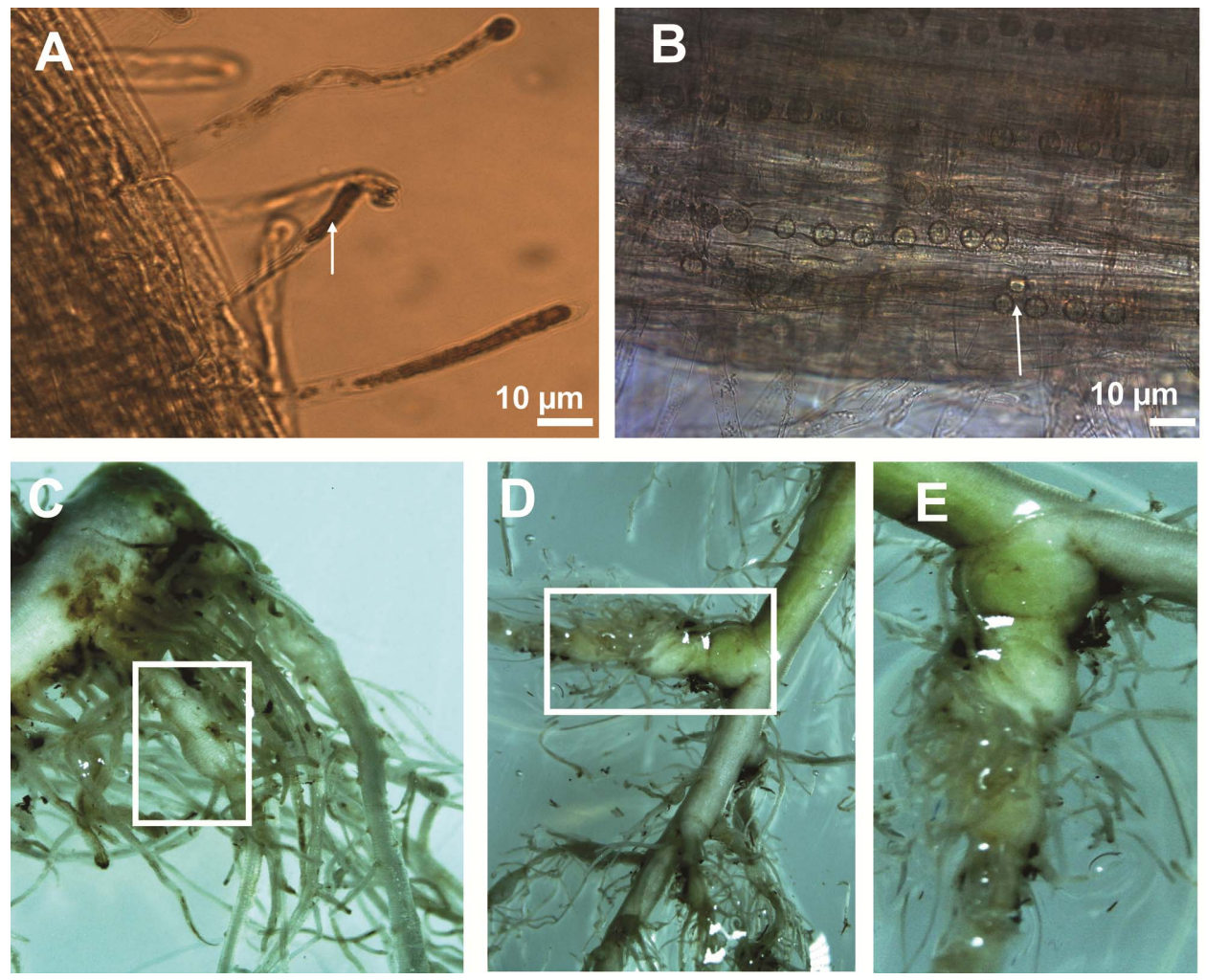

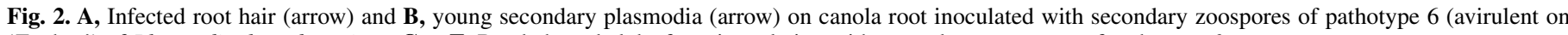
'Zephyr') of Plasmodiophora brassicae. C to E, Bead-shaped clubs from inoculation with secondary zoospores of pathotype 6. 

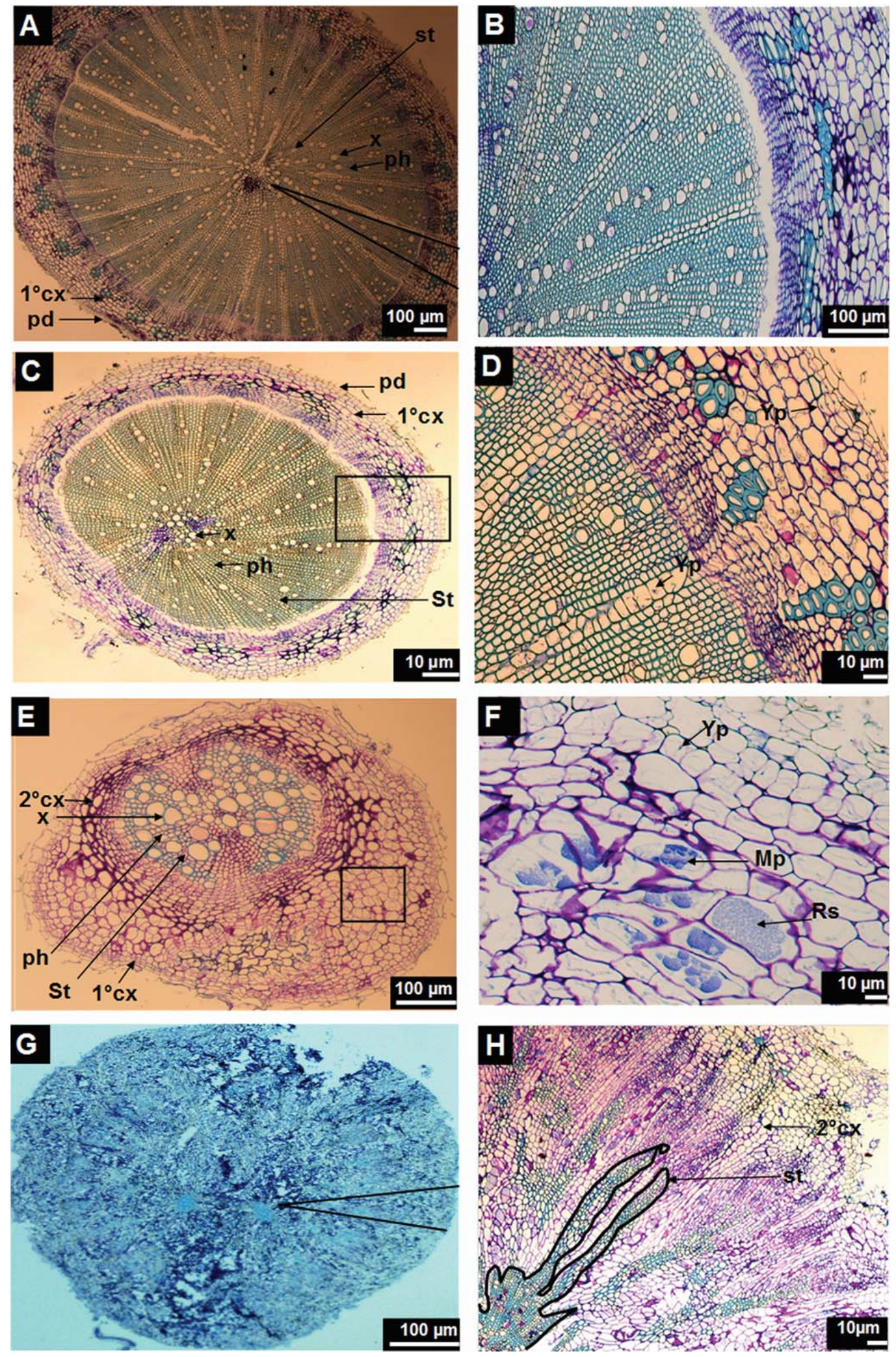

Fig. 3. Cross-section of roots of healthy and Plasmodiophora brassicae-infected canola plants, stained with toluidine blue O. A, Healthy root (control). Lignified xylem cell walls stain dark blue, nonlignified walls are pink. B, Magnified region from A showing intact stele, cortex, and periderm. C, Roots of plants inoculated with resting spores of pathotype 6 (avirulent on 'Zephyr'). D, Magnified region from C showing intact stele, cortex, and periderm (young plasmodia are marked with arrow). E, Bead-shaped clubs produced after inoculation with secondary zoospores of pathotype 6 . Note the proliferation of cortical tissue outside of the stele, and no proliferation within the stele. F, Magnified region from E showing young plasmodia, mature plasmodia, and resting in the cortical cells. G, Typical clubs. Stele region is disrupted. $\mathbf{H}$, Magnified region from $\mathrm{G}$ showing invasion and expansion of stele (marked with black boundaries). Annotations: $\mathrm{x}=\mathrm{xylem}$, $\mathrm{ph}=$ phloem, $1^{\circ} \mathrm{cx}=$ primary cortex, $2^{\circ} \mathrm{cx}=$ secondary cortex, $\mathrm{pd}=$ periderm, $\mathrm{St}=$ stele, $\mathrm{Yp}=$ young plasmodia, $\mathrm{Mp}=$ mature plasmodia, and $\mathrm{Rs}=$ resting spores . 
zoosporangium $(n=20)$ and the nuclear size varied from 1.0 to $1.5 \mu \mathrm{m}(n=50)$ (Fig. 4A).

The flagellate SZ collected in deionized water had a mean length of $14.4 \pm 0.19 \mu \mathrm{m}$ and width of $9.6 \pm 0.32 \mu \mathrm{m}(n=55)$, and all had two anterior whiplash flagella (Fig. 4B). The mean diameter of encysted zoospores (Fig. 4D) obtained by centrifugation was $21.7 \pm 0.37 \mu \mathrm{m}(n=50)$. All of the flagellate and encysted zoospores were uninucleate (Fig. 4C and D). SZ that were released into phosphate buffer or physiological saline were slightly smaller than those produced in deionized water. The length and width of flagellate zoospores in phosphate buffer were $12.5 \pm$ 0.23 by $8.7 \pm 0.25 \mu \mathrm{m}$, respectively; and, in physiological saline, were $11.8 \pm 0.29$ by $8.3 \pm 0.30 \mu \mathrm{m}$, respectively. The diameters of encysted zoospores in phosphate buffer and physiological saline were $17.8 \pm 0.28$ and $17.3 \pm 0.44 \mu \mathrm{m}$, respectively.

\section{DISCUSSION}

This is the first study to compare the roles of primary and secondary infection in both compatible and incompatible reactions between $P$. brassicae and a host. We provide evidence that RHI by $P$. brassicae plays an important role in host recognition of the pathogen and influences the development of the pathogen at the cortical infection stage. Zephyr canola was chosen because it is susceptible to P3 (compatible reaction) and resistant to P6 (incompatible reaction); these reactions were consistent across the trials. The severity of clubroot symptoms (DSI) exhibited the same pattern of response as the extent of secondary infection of the root cortex and the number of cells with RS; therefore, these are the assessments that the discussion will focus on

Inoculation with RS-P6 (RS of P6, avirulent on Zephyr) resulted in no symptoms and very limited development of the pathogen, as expected in an incompatible interaction. Inoculation with RS-P3 (RS of P3, virulent on Zephyr) resulted in the greatest symptom development and highest number of cortical cells containing RS. This was as expected in a compatible reaction $(6,12,17)$. Inoculation with SZ of P6 alone (SZ-P6) resulted in much more cortical infection than with RS-P6, while inoculation with SZ-P3 alone resulted in less cortical infection than RS-P3. Comparison of the effect of inoculation with RS versus SZ on cortical infection and DSI provided a measure of the role of primary infection in initiation of resistance or susceptibility. These results demonstrate that primary infection plays a role in subsequent cortical infection in both compatible and incompatible reactions. Cortical infection was almost completely suppressed after primary infection with an avirulent pathotype (RS-P6) but the root cortex was infected and colonized to a substantial extent with SZ of an avirulent pathotype (SZ-P6). This indicates that primary infection with P6 induced a resistance response that was most strongly expressed in the root cortex, although there was also a small effect on pathogen development in root hairs. Conversely, cortical infection following primary infection with a virulent pathotype (RS-P3) was more extensive than with SZ of a virulent pathotype (SZ-P3) alone, which indicates that primary infection induced susceptibility or suppressed resistance in the root cortex.

Comparison of RS-P6 + SZ-P3 versus RS-P3 + SZP3 or SZ-P3 provided a measure of the impact of early initiation of a resistance reaction on subsequent development of a virulent pathotype. Inoculation with RS-P6 + SZ-P3 resulted in less infection and lower severity than RS-P3 + SZP3 or SZ-P3. This response provides additional support for the hypothesis that primary infection has a role in inducing resistance in an incompatible reaction. In fact, it provides evidence that primary infection is an important step in recognition of the avirulent pathogen. Similarly, comparison of RS-P3 versus RS-P3 + SZ-P3 or SZ-P3 alone
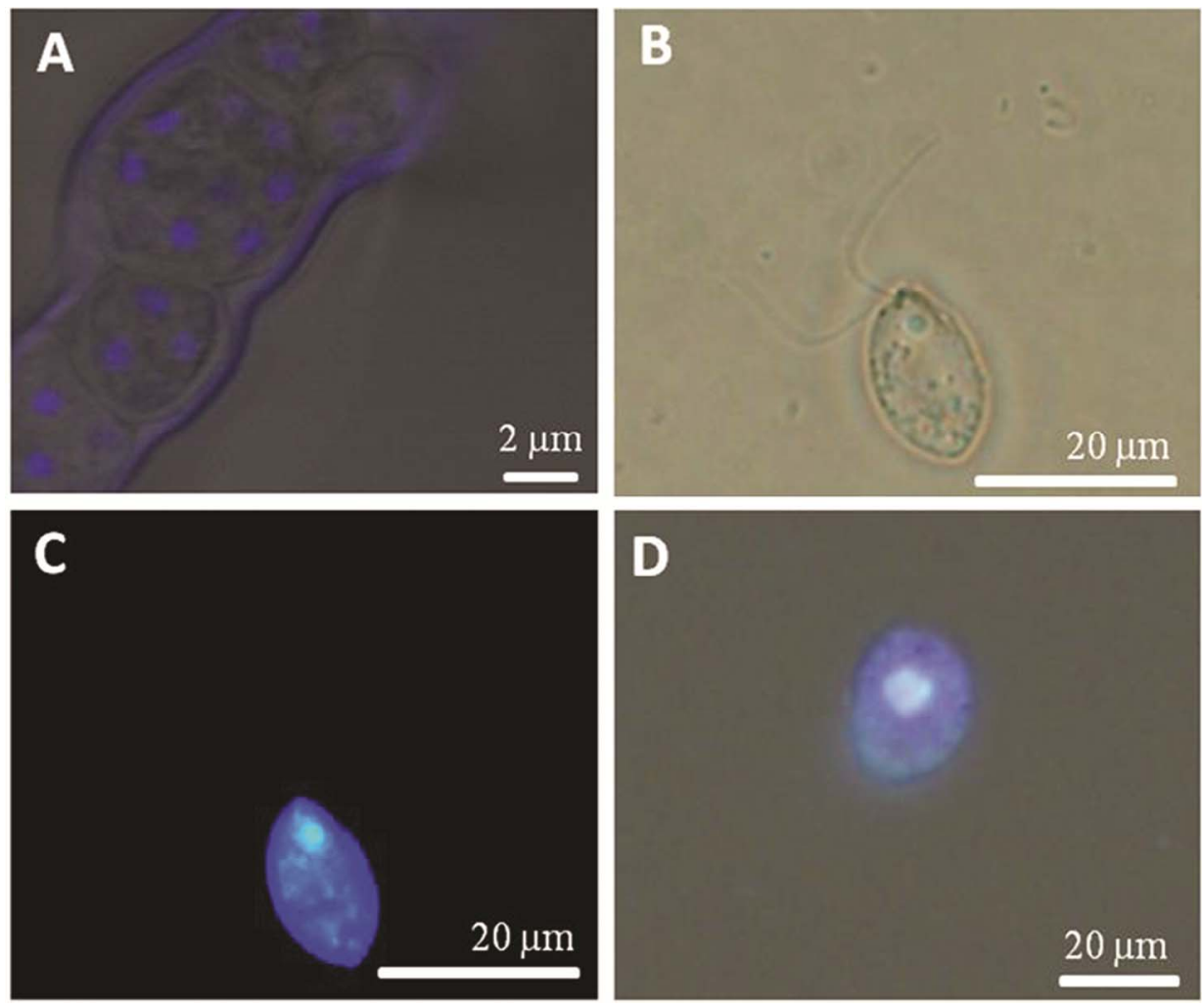

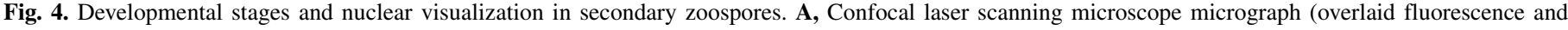

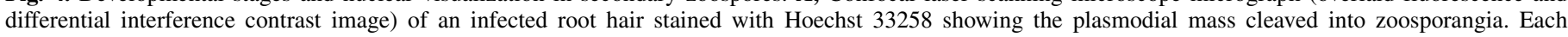

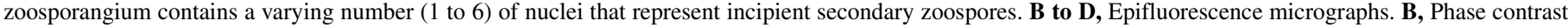

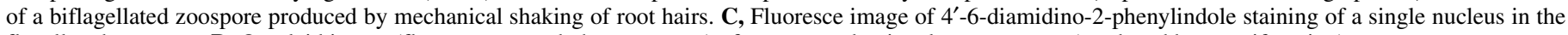
flagellated zoospore. D, Overlaid image (fluorescence and phase contrast) of an encysted uninucleate zoospore (produced by centrifugation). 
provided a measure of the impact of early suppression of resistance on subsequent development of a virulent pathotype in the compatible reaction. Inoculation with RS-P3 produced more cortical infection and higher severity than SZ-P3 alone but the same level of symptom expression and cortical infection as RS-P3 + SZ-P3. This indicates that primary infection results in a slight but potentially important suppression of host defenses in a susceptible host.

Inoculation with SZ-P6 resulted in infection of the root cortex and pathogen development sufficient to produce viable RS. However, symptom development was restricted to small, beadlike clubs. This indicates that, if recognition of the avirulent pathotype does not occur at the primary infection stage, initiation of a resistance response was delayed but not completely suppressed.

Inoculation with RS-P3 and RS-P3 + SZ-P3 resulted in the maximum possible level of clubroot severity (100 DSI) and the greatest area of cortical infection, which might represent the maximum that can occur in this host-pathogen interaction. The initial inoculation with $5 \mathrm{ml}$ of RS at $3 \times 10^{5} \mathrm{ml}^{-1}$ may have been sufficient to produce 100 DSI. Indeed, there are other reports of $100 \%$ clubroot severity with similar $\left(5 \mathrm{ml}\right.$ of $\left.1 \times 10^{6} \mathrm{ml}^{-1}\right)$ concentrations of inoculum $(7,9)$. Repeating this comparison with a lower inoculum concentration might help to elucidate the role of primary infection in a compatible interaction.

The only result that did not support a role for primary infection in the initiation of a resistance reaction in the host was the comparison of RS-P6 + SZ-P3 versus SZ-P3. If primary infection by an avirulent pathotype stimulates the early initiation of host resistance, inoculation with RS-P6 before adding SZ-P3 would be expected to suppress infection and symptom development compared with SZ alone. However, the reverse was observed. There was slightly more cortical infection with RS-P6 + SZ-P3 than with SZ-P3 alone (18 versus $12 \%$ ), more cells with RS (15 versus 12 cells) and higher clubroot severity (84 versus 67 DSI). This may indicate that primary infection has a larger role in suppressing host defenses than in stimulating resistance. However, additional work in this area is required.

Most physiological and molecular studies on clubroot have focused only on the compatible relationship, and indicate that fewer physiological changes occur in the host during the primary phase of the pathogen's lifecycle compared with the secondary stage $(4,41)$. However, none of the effectors that mediate compatible and incompatible relationships with $P$. brassicae have been identified or characterized. During infection in many host-pathogen systems, the pathogen secretes effectors that interact with the host and play an important role in virulence or the stimulation of a resistance (incompatible) response $(16,20,26,28)$. To establish a successful infection, $P$. brassicae must either suppress host resistance or fail to trigger host resistance. In either case, the response is likely triggered by effectors. In nonhosts, $P$. brassicae triggered a resistance response (33) but RHI was critical for induction of resistance to secondary infection and subsequent club formation (14).

In a recent study of gene expression in primary zoospores and SZ of $P$. brassicae, many more genes were upregulated in primary zoospores than in SZ (13). The authors suggested that the genes that were upregulated in SZ likely contribute to infection of cortical tissue. The results of the present study support their hypothesis that there are specific mechanisms required for secondary infection, in that SZ can infect the root cortex in the absence of primary infection but primary infection has an important role in the recognition of a compatible or incompatible pathotype.

The results of the current study are also consistent with the results of a study conducted to identify changes in host gene expression in Arabidopsis thaliana (L.) Heynh inoculated with the compatible $P$. brassicae ecotype Co-1 (1). At 4 days after inoculation (which corresponds to primary infection), expression of several genes known to be involved in pathogen recognition and signal transduction was induced. Also, many more host genes were downregulated than were upregulated. Some of the downregulated genes were involved in lignin and salicylic acid biosynthesis, the oxidative burst pathway, and several other defenserelated genes. In general, these results support the hypothesis that primary infection suppresses host resistance. They are also consistent with a recent study that identified that a compatible $P$. brassicae-canola interaction is characterized by a lack of the reactive oxygen species and a reduction in lignin relative to an incompatible interaction (6).

Infection and development of $P$. brassicae in the root cortex of susceptible and resistant cultivars has been studied in detail $(6,7,11,17,19,30)$. Clubroot resistance was expressed most clearly and consistently in the root cortex, where pathogen development occurred quickly in susceptible cultivars. Very little or no pathogen development was observed in highly resistant radish (30) and canola cultivars (6). In the current study, plasmodia were observed in the root cortex of plants inoculated with RS of the avirulent pathotype, but these did not develop to produce RS and no clubs developed on the roots. This is consistent with recent studies showing that resistance can be expressed after some initial colonization of the root cortex in canola (7) and cabbage (17).

The present study provides some insights into the timing of the interaction between effectors from $P$. brassicae and the host. Plants inoculated with RS of the avirulent pathotype (P6) did not develop clubs, whereas direct inoculation with the SZ of P6 resulted in infection and development of small bead-shaped clubs. This indicates that the initial recognition of the pathogen normally occurs during primary infection, so that the host is able to respond with effective resistance mechanisms when challenged by SZ. Direct inoculation with SZ either bypassed this recognition stage or did not provide enough time for the resistance reaction to develop fully. However, the results of this study also showed that recognition of the pathogen can occur during cortical infection and subsequent development of the pathogen. Inoculation with SZ of the incompatible pathotype (P6) resulted in lower levels of pathogen development than inoculation with SZ of the compatible pathotype (P3) which, in turn, was lower than from inoculation with RS of P3.

The small, bead-shaped clubs produced by SZ of P6 in this study appear to be similar to the spheroid galls reported in resistant reactions by Williams (45) and others $(29,37)$. There is some disagreement in the literature as to whether this is a true resistance response. Rennie et al. (37) point out that several researchers considered these an indication of host resistance because the spheroid clubs (galls) represent a restriction of the pathogen within the host. However, the authors suggest that the formation of spheroid clubs should not be interpreted as complete resistance, because small numbers of RS can develop in these clubs. The results of the current study support the conclusion that this reaction does not represent complete resistance. Instead, small clubs are produced when pathogen development is limited after some development has already occurred in the root cortex. This, in turn, indicates that some components of host resistance were bypassed or delayed. Similarly, bead-shaped clubs were observed on canola inoculated with SZ produced on a nonhost (14). This further supports the hypothesis that direct infection by SZ bypasses recognition by the host during primary infection. However, clubbing symptoms do not develop fully, likely because initiation of resistance is delayed or effectors at the primary infection stage are required to allow a fully compatible interaction.

Differences in the incidence and development of primary infection of resistant and susceptible host cultivars have been studied in detail $(6,11)$. The results of the current study support 
the observation that both virulent and avirulent pathotypes can infect root hairs but that the incidence of primary infection is higher in plants inoculated with virulent pathotypes than with the avirulent pathotypes $(6,22)$. The present study also confirms previous reports that $\mathrm{SZ}$ of $P$. brassicae can initiate both primary and secondary infection $(12,14,35)$.

SZ were examined for the presence of binucleate zoospores because there were reports that binucleate zoospores of $P$. brassicae result from fusion of two zoospores $(5,44)$ and that cortical infection occurs after the SZ fuse in pairs $(10,24)$. In the current study, all of the SZ were uninucleate, which indicates that the majority of SZ do not fuse. Further study into this phase of the life cycle is warranted. If fusion of SZ was a prerequisite to cortical infection, this would greatly reduce the effective inoculum concentration. The SZ in this study were morphologically identical to primary zoospores (oval shaped, with one nucleus and two flagella of uneven length), as has been reported in previous studies $(12,14,27)$.

The average size of SZ released in deionized water after mechanical shaking of the roots was 9.6 by $14.4 \mu \mathrm{m}$, while those released into phosphate buffer were slightly smaller. SZ observed within root hairs were reported to be much smaller, 2.5 by $3.5 \mu \mathrm{m}$ (24) or 1.5 by 0.5 to $0.7 \mu \mathrm{m}$ (5). Differences in the size of SZ were also observed when they were switched from $10 \%$ glucose solution to water (J. Feng, unpublished data). Because the zoospores in the present study were released in deionized water, it is highly likely that they would take up more water and swell to a larger size than those in a buffer or an ionized solution. SZ within a root hair are likely smaller than released zoospores as a result of higher osmotic or physical pressure in the root hairs. This is the first report of the size of free-living SZ.

The methods used to produce and collect SZ were those of Feng et al. (14). This method has several advantages over using infected plants as a source of SZ, because the inoculum concentration can be quantified and adjusted as required. However, it proved to be difficult to consistently obtain large quantities of SZ; therefore, infected seedlings were used as a source of SZ (35) for some of the studies. The results using this approach were consistent between repetitions of the study and exhibit a pattern of response similar to that of studies using collected SZ; thus, we conclude that this is a suitable inoculation approach for use in future studies.

In summary, this is the first study to demonstrate that the primary infection phase of $P$. brassicae plays an important role in host recognition of the pathogen. The results support the hypothesis that primary infection suppresses the initiation of resistance in a susceptible cultivar and also stimulates the resistance reaction in a resistant cultivar. It appears likely that pathogen effectors are recognized at the primary infection stage and that this early interaction between host and pathogen contributes to the resistant or susceptible reaction in the host at the secondary infection stage. Induction of susceptibility was more effective than initiation of resistance in the two pathotype-host interactions examined. Recognition of the pathogen as compatible or incompatible also occurred in the root cortex but the resistance reaction developed more quickly and was expressed more strongly in response to primary infection. Our results were consistent with independent studies of gene expression in the pathogen and host in a compatible interaction. However, additional study at the molecular level is needed to further elucidate the role of primary zoospores and $\mathrm{SZ}$ in pathogenesis.

\section{ACKNOWLEDGMENTS}

We thank the Clubroot Risk Mitigation Initiative of Agriculture and Agri-Food Canada and the Canola Council of Canada for financial support, and the Animal Health Lab, University of Guelph, for root section preparation and staining.

\section{LITERATURE CITED}

1. Agarwal, A., Kaul, V., Faggaian, R., Rookes, J. E., Ludwig-Muller, J., and Cahill, D. 2011. Analysis of global host gene expression during the primary phase of the Arabidopsis thaliana-Plasmodiophora brassicae interaction. Funct. Plant Biol. 38:462-478.

2. Agrios, G. N. 2005. Plant Pathology. Elsevier Academic Press, Amsterdam.

3. Cao, T., Manolii, V. P., Hwang, S. F., Howard, R. J., and Strelkov S. E. 2009. Virulence and spread of Plasmodiophora brassicae (clubroot) in Alberta, Canada. Can. J. Plant Pathol. 31:321-329.

4. Cao, T., Srivastava, S., Rahman, M. H., Kav, N. N. V., Hotte, N., Deyholos, M. K., and Sterelkov, S. E. 2008. Proteome-level changes in roots of Brassica napus as a result of Plasmodiophora brassicae infection. Plant Sci. 174:97-115.

5. Cook, W. R. I., and Swartz, E. J. 1930. The life-history, cytology and method of infection of Plasmodiophora brassicae Wor., the cause of the finger-and-toe disease of cabbages and other crucifers. Philos. Trans. R. Soc. Lond. 218:283-314.

6. Deora A., Gossen B. D., McDonald M. R. 2012. Infection and development of Plasmodiophora brassicae in resistant and susceptible canola cultivars. Can. J. Plant Pathol. 34:239-247. doi:10.1080/ 07060661.2012 .681071

7. Deora, A., Gossen, B. D., and McDonald, M. R. 2013. Cytology of infection, development, and expression of resistance to Plasmodiophora brassicae in canola. Ann. Appl. Biol. 163:56-71. doi:10.1111/aab.12033

8. Diederichsen, E., Frauen, M., Linders, E. G. A., Hatakeyama, K., and Hirai, M. 2009. Status and perspectives of clubroot resistance breeding in crucifer crops. J. Plant Growth Regul. 28:265-281.

9. Dixon, G. R. 2009. Plasmodiophora brassicae in its environment. J. Plant Growth Regul. 28:212-228.

10. Dobson, R. L., and Gabrielson, R. L. 1983. Role of primary and secondary zoospores of Plasmodiophora brassicae in the development of clubroot in Chinese cabbage. Phytopathology 73:559-561.

11. Donald, E. C., Jaudzems, G., and Porter, I. J. 2008. Pathology of cortical invasion by Plasmodiophora brassicae in clubroot resistant and susceptible Brassica oleracea hosts. Plant Pathol. 57:201-209.

12. Feng, J., Hwang, S. F., and Strelkov, S. E. 2013. Studies into primary and secondary infection processes by Plasmodiophora brassicae on canola. Plant Pathol. 62:177-183.

13. Feng, J., Hwang, S. F., and Strelkov, S. E. Assessment of gene expression profiles in primary and secondary zoospores of Plasmodiophora brassicae by dot blot and real-time PCR. Microbiol. Res. In press. doi:10.1016/j.micres.2013.02.011

14. Feng, J., Xiao, Q., Hwang, S. F., Strelkov, S. E., and Gossen, B. D. 2012. Infection of canola by secondary zoospores of Plasmodiophora brassicae produced on a non-host. Eur. J. Plant Pathol. 132:309-315.

15. Friberg, H., Lagerlof, J., and Ramert, B. 2006. Usefulness of non-host plants in managing Plasmodiophora brassicae. Plant Pathol. 55:690-695.

16. Gan, P. H. P., Rafiqi, M., Hardham, A. R., and Dodds, P. N. 2010. Effectors of biotrophic fungal plant pathogens. Funct. Plant Biol. 37:913918.

17. Gludovacz, T. V. 2013. Clubroot in canola and cabbage in relation to soil temperature, plant growth and host resistance. M.Sc. thesis, University of Guelph, Guelph, ON, Canada.

18. Gossen, B. D., McDonald, M. R., Hwang, S. F., Strelkov, S. E., and Peng, G. 2013. Comparison of clubroot (Plasmodiophora brassicae) development and management on canola and Brassica vegetables. Can. J. Plant Pathol. 35:175-191.

19. Gustafsson, M., Liljeroth, E., Gunnarsson, M., and Lundborg, T. 1986. Effects of infection by Plasmodiophora brassicae on root anatomy of rape. J. Phytopathol. 117:144-151.

20. Hoghenhout, S. A., Van der Hoorn, R. A., Terauchi, R., and Kamoun, S. 2009. Emerging concepts in effector biology of plant-associated organisms. Mol. Plant-Microbe Interact. 22:115-122.

21. Howard, R. J., Strelkov, S. E., and Harding, M. W. 2010. Clubroot of cruciferous crops-new perspective on an old disease. Can. J. Plant Pathol. 32:43-57.

22. Hwang, S. F., Ahmed, H. U., Zhou, Q., Strelkov, S. E., Gossen, B. D., Peng, G., and Turnbull, G. D. 2012. Assessment of the impact of resistant and susceptible canola on Plasmodiophora brassicae inoculum potential. Plant Pathol. 61:945-952. doi:10.1111/j.1365-3059.2011.02582.x

23. Hwang, S. F., Strelkov, S. E., Feng, J., Gossen, B. D., and Howard, R. J. 2012. Plasmodiophora brassicae: A review of an emerging pathogen of the Canadian canola (Brassica napus) crop. Mol. Plant Pathol. 13:105113.

24. Ingram, D. S., and Tommerup, C. 1972. The life history of Plasmodiophora brassicae Woron. Proc. R. Soc. Lond. Ser. B 180:103-112.

25. Jones, D. R., Ingram, D. S., and Dixon, G. R. 1982. Characterization of isolates derived from single resting spores of Plasmodiophora brassicae 
and studies of their interaction. Plant Pathol. 31:239-246.

26. Jones, J. D., and Dangl, J. L. 2006. The plant immune system. Nature 444:323-329.

27. Kageyama, K., and Asano, T. 2009. Life cycle of Plasmodiophora brassicae. J. Plant Growth Regul. 28:203-211.

28. Kamoun, S. 2006. A catalogue of the effector secretome of plant pathogenic oomycetes. Annu. Rev. Phytopathol. 44:41-60.

29. Kobelt, P., Siemens, J., and Sacristán, M. D. 2000. Histological characterization of the incompatible interaction between Arabidopsis thaliana and the obligate biotrophic pathogen Plasmodiophora brassicae. Mycol. Res. 2:220-225.

30. Kroll, T. K., Lacy G.H., and Moore L. D. 1983. A quantitative description of the colonization of susceptible and resistant radish plants by Plasmodiophora brassicae. J. Phytopathol. 108:97-105.

31. Kuginuki, Y., Yoshikawa, H., and Hirai, M. 1999. Variation in virulence of Plasmodiophora brassicae in Japan tested with clubroot-resistant cultivars of Chinese cabbage (Brassica rapa L. ssp. pekinensis). Eur. J. Plant Pathol. 105:327-332.

32. LeBoldus, J. M., Manolii, V. P., Turkington, K. T., and Sterlkov, S. E. 2012. Adaptation to Brassica host genotypes by a single-spore isolate and population of Plasmodiophora brassicae (clubroot). Plant Dis. 96:833838.

33. Ludwig-Muller, J., Ihmig, S., Bennett, R., Kiddle, G., Ruppel, M., and Hilgenberg, W. 1999. The host range of Plasmodiophora brassicae and its relationship to endogenous glucosinolate content. New Phytol. 141:443458.

34. Macfarlane, I. 1952. Factors affecting the survival of Plasmodiophora brassicae Wor. in the soil and its assessment by a host test. Ann. Appl. Biol. 39:239-256

35. Naiki, T., Kawaguchi, C., and Ikegami, H. 1984. Root hair reinfection in Chinese cabbage seedlings by the secondary zoospores of Plasmodiophora brassicae Woronin. Ann. Phytopathol. Soc. Jpn. 50:216220 .
36. Peterson, R. L., Peterson, C. A., and Melville, L. H. 2008. Teaching Plant Anatomy Through Creative Exercises. NRC Press, Ottawa, ON, Canada.

37. Rennie, D. C., Manolii, V. P., Plishka, M., and Strelkov, S. E. Histological analysis of spindle and spheroid root galls caused by Plasmodiophora brassicae. Eur. J. Plant Pathol. In press. doi:10.1007/s10658-012-0119-x

38. Sharma, K., Gossen, B. D., Greenshields, D., Selvaraj, G., Strelkov, S. E., and McDonald, M. R. 2013. Reaction of lines of the Rapid Cycling Brassica Collection and Arabidopsis thaliana to selected pathotypes of Plasmodiophora brassicae. Plant Dis. 97:720-727.

39. Sharma, K., Gossen, B. D., and McDonald, M. R. 2011. Effect of temperature on primary infection by Plasmodiophora brassicae and initiation of clubroot symptoms. Plant Pathol. 60:830-838.

40. Sharma, K., Gossen, B. D., and McDonald, M. R. 2011. Effect of temperature on cortical infection by Plasmodiophora brassicae and clubroot severity. Phytopathology 101:1424-1432.

41. Siemens, J., Keller, I., and Sarx, J. 2006. Transcriptome analysis of Arabidopsis clubroots indicate a key role for cytokinins in disease development. Mol. Plant-Microbe. Interact. 19:480-494.

42. Strelkov, S. E., Manolii, V. P., Cao, T., Xue, S., and Hwang, S. F. 2007. Pathotype classification of Plasmodiophora brassicae and its occurrence in Brassica napus in Alberta, Canada. J. Phytopathol. 155:706-712.

43. Strelkov, S. E., Tewari, J. P., Smith, E., and Smith-Degenhardt, E. 2006 Characterization of Plasmodiophora brassicae populations from Alberta, Canada. Can. J. Plant Pathol. 28:467-474.

44. Tommerup, I. C., and Ingram, D. S. 1971. The life history of Plasmodiophora brassicae Woron. in Brassica tissue cultures and in intact roots. New Phytol. 70:327-332.

45. Williams, P. H. 1966. A system for the determining of races of Plasmodiophora brassicae that infect cabbage and rutabaga. Phytopathology 56:624-626.

46. Xue, S., Cao, T., Howard, R. J., Hwang, S. F., and Strelkov, S. E. 2008. Isolation and variation in virulence of single-spore isolates of Plasmodiophora brassicae from Canada. Plant Dis. 92:456-462. 